\title{
Datación por ESR del yacimiento arqueológico del Pleistoceno inferior de Vallparadís (Terrassa, Cataluña, España)
}

\author{
ESR dating of the Early Pleistocene archaeological site at Vallparadís (Terrassa, \\ Cataluña, Spain)
}

\author{
Mathieu Duval (*) \\ Davinia Moreno $(* *)(* * *)$ \\ Qingfeng Shao (**) \\ Pierre Voinchet $(* *)$ \\ Christophe Falguères (**)
}

\author{
Jean-Jacques Bahain (**) \\ Tristan Garcia $(* * * *)$ \\ Joan García $(* * *)$ \\ Kenneth Martínez $(* * *)$
}

\begin{abstract}
RESUMEN
Excavado entre 2005 y 2007, el yacimiento de Vallparadís (Terrassa, España) ha aportado una fauna rica y diversa en asociación con un importante conjunto lítico de Modo 1. Con el objetivo de completar el marco cronoestratigráfico, se hicieron dataciones por los métodos de Resonancia Paramagnética Electrónica (Electron Spin Resonance, ESR) y ESR combinada con uranio-torio sobre algunas muestras de granos de cuarzo extraídos de sedimentos y dientes fósiles, respectivamente. Los resultados obtenidos son muy significativos, ofreciendo edades ESR promedias de $0,83 \pm 0,13 \mathrm{Ma}(2 \sigma)$ para el nivel arqueológico de referencia del yacimiento (nivel 10) y de $0,79 \pm 0,23 \mathrm{Ma}(2 \sigma)$ para el nivel arqueológico 12 de la secuencia de Vallparadís. Dichos resultados por ESR concuerdan con los obtenidos a partir de la biocronología y del paleomagnetismo. El yacimiento de Vallparadís dispone ahora de un marco cronoestratigráfico fiable y coherente, que permite situarlo cronológicamente a finales del Pleistoceno inferior, dentro de un rango temporal comprendido entre los eventos geomagnéticos de Jaramillo y Brunhes. Dicho yacimiento junto a los de Gran Dolina TD-6 y Sima del Elefante TE-9, en Atapuerca, Burgos, se posiciona como un yacimiento clave para el estudio de los primeros poblamientos humanos de Europa.
\end{abstract}

\begin{abstract}
Excavated between 2005 and 2007, the site of Vallparadis (Terrassa, Spain) has given an abundant and diversified fauna associated with a rich Mode 1 industry. In
\end{abstract}

(*) Centro Nacional de Investigación sobre la Evolución Humana (CENIEH). Paseo de Atapuerca s/n. 09002 Burgos. España. Correo e.: mathieu.duval@cenieh.es order to complete the chronostratigraphic framework, we performed ESR and combined US-ESR dating applied to quartz grains extracted from sediments and fossil teeth, respectively. The results obtained are very significant, giving a weighted mean ESR age of $0.83 \pm 0.13 \mathrm{Ma}(2 \sigma)$ for the reference archaeological level of the site (level 10) and another of $0.79 \pm 0.23 \mathrm{Ma}(2 \sigma)$ for the archaeological level 12 of the Vallparadis sequence. These ESR results are in total agreement with the ones derived from biochronology and paleomagnetism. This whole dataset allows the elaboration of a reliable and consistent chronostratigraphic framework which chronologically places Vallparadis site in the late Early Pleistocene period, i.e. within a time range comprised between Jaramillo and Brunhes geomagnetic events. Consequently, as well as Gran Dolina-TD6 and Sima del Elefante- TE9 sites, in Atapuerca, Burgos, Vallparadis can be therefore considered as a key site for the study of early hominid settlements in Europe.

Palabras clave: Península Ibérica; Colonización de Eurasia; Industria Modo 1; Geocronología; Datación por Resonancia Paramagnética Electrónica; Uranio-torio; Dientes fósiles.

(**) Muséum National d'Histoire Naturelle, Département de Préhistoire, UMR7194 du CNRS. 1 rue R. Panhard. 75013 Paris. Francia. Correos e.: moreno@mnhn.fr;

qingfengshao@hotmail.com; pvoinch@mnhn.fr; falguere@mnhn.fr; bahain@mnhn.fr

$(* * *)$ Área de Prehistoria - Institut Català de Paleoecologia Humana i Evolució Social (IPHES). Universitat Rovira i Virgili. Pza. Imperial Tarraco 1. 45003 Tarragona. España. Correos e.: davinia.moreno@urv.cat; jgarcia@prehistoria.urv.cat; kenneth@prehistoria.urv.cat

(***) CEA, LIST, Laboratoire National Henri Becquerel (LNE LNHB). 91191 Gif-sur-Yvette. Francia. Correo e.: tristan.garcia@cea.fr

Recibido: 12-IV-2010; aceptado: 16-VI-2010. 
Key words: Iberian Peninsula; Early hominid settlements in Eurasia; Mode 1 industry; Geochronology; ESR dating; Uranium-Thorium; Fossil teeth.

\section{INTRODUCCIÓN}

Desde hace algunas décadas, numerosas evidencias de ocupación humana antigua han sido descubiertas a lo largo del continente europeo. Esto ha permitido empezar a establecer un marco cronoestratigráfico y cultural relativamente fiable de la primera expansión humana europea (Carbonell y Rodríguez 2006; Lumley et al. 2009). La mayoría de los yacimientos arqueológicos se localizan en la cuenca mediterránea, en Francia (Pont-de-Lavaud, Despriée et al. 2006; cueva de Vallonnet, Lumley et al. 1988), en Italia (Monte Poggiolo, Peretto et al. 1998), en España (Gran Dolina, Parés y Pérez-González 1999; Sima del Elefante, en Atapuerca, Carbonell et al. 2008; Barranco León y Fuente Nueva-3, Martínez-Navarro et al. 1997; Oms et al. 2000) y en Georgia (Dmanisi, Lordkipanidze et al. 2007). Descubiertos en los años 1980-1990, dichos yacimientos han proporcionado tanto fósiles humanos como testimonios de actividades humanas (industria lítica, marcas de corte sobre huesos), lo cual ha permitido retrasar la llegada del género Homo en Europa al Pleistoceno inferior. Además de estos yacimientos "históricos", considerados hoy como referentes, se descubrieron más recientemente otros con posibles trazas de ocupaciones humanas y cuyos primeros estudios refuerzan la hipótesis de una colonización antigua de Europa: Pirro Nord (Italia, Arzarello et al. 2007), Lézignan-la-Cèbe (Francia, Crochet et al. 2009), Lunery (Francia, Despriée et al. 2007, 2010) y Kozarnika (Bulgaria, Guadelli et al. 2005).

Vallparadís (Terrassa, Cataluña) (Fig. 1), descubierto en 2005 durante las obras de construcción de una estación de tren, pertenece a este último conjunto de nuevos yacimientos. Los primeros resultados obtenidos a partir del estudio de la fauna y del paleomagnetismo han mostrado su antigüedad. Con el objetivo de completar el marco cronoestratigráfico preliminar establecido a partir de estos trabajos, era necesaria la aplicación de un método de datación absoluta. Debido al contexto geológico, el material disponible in situ y el rango de tiempo estudiado, el método

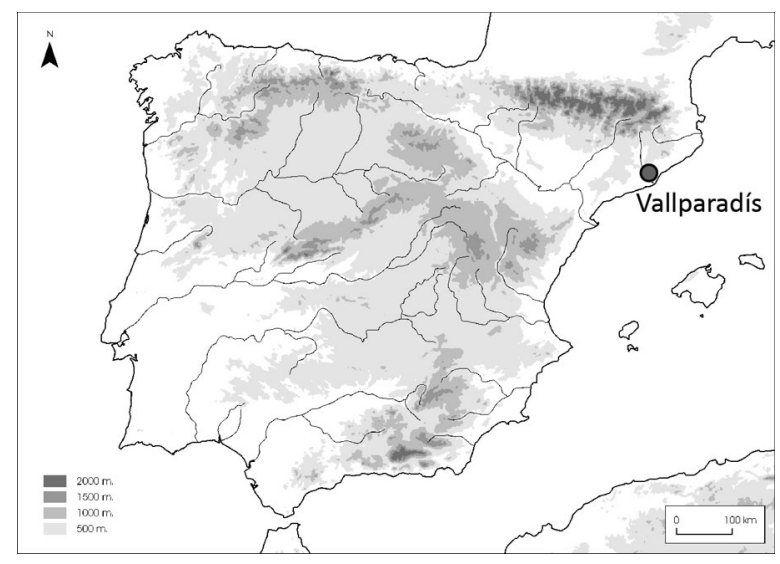

Fig. 1. Yacimiento del Pleistoceno inferior de Vallparadís (Terrassa, Barcelona): situación geográfica.

de Resonancia Paramagnética Electrónica (1) forma parte de los pocos métodos potencialmente aplicables, junto con la luminiscencia (termoluminiscencia (2), luminiscencia ópticamente estimulada (3), luminiscencia estimulada por infrarrojos (4), cuyos límites de aplicación han sido ampliados recientemente (ejemplos en Rhodes et al. 2006; Berger et al. 2008), y los métodos basados en la detección de isótopos cosmogénicos (5) (Granger y Muzikar 2001).

El método ESR tiene la ventaja de ser aplicable a una gran variedad de materiales (Ikeya 1993) que permiten así cubrir todos los contextos sedimentarios posibles, lo que le convierte en el método más usado en la datación de los yacimientos europeos antiguos: espeleotemas (cueva de Vallonnet, Yokoyama et al. 1988), granos de cuarzo sedimentario (Pont-de-Lavaud y Lunery, Voinchet et al. 2010; Monte Poggiolo, Peretto et al. 1998; Fuente Nueva-3 y Barranco León (6)) o dientes fósiles (Gran Dolina, Falguères et al. 1999; Orce (7); Dmanisi (8)). En el yacimien-

(1) Electron Spin Resonance (ESR).

(2) Thermoluminescence (TL).

(3) Optically Stimulated Luminescence (OSL).

(4) Infrared Stimulated Luminescence (IRSL).

(5) Terrestrial in situ Cosmogenic Nuclides (TCN).

(6) Duval, M. 2008: Évaluation du potentiel de la méthode de datation par Résonance de Spin Electronique (ESR) appliquée aux gisements du Pléistocène inférieur: étude des gisements d'Orce (bassin de Guadix-Baza, Espagne) et contribution à la connaissance des premiers peuplements de l'Europe. Tesis de doctorado no editada. Muséum National d'Histoire Naturelle. Paris.

(7) Véase nota 6.

(8) Garcia, T. 2004: Cadre stratigraphique, magnétostra- 
to de Vallparadís, el método ESR ha sido aplicado a dos tipos de materiales: dientes de équidos (datos ya publicados en Martínez et al. 2010) y granos de cuarzo extraídos de sedimentos fluviales.

\section{EL YACIMIENTO DE VALLPARADÍS (EVT)}

Entre 2005 y 2007, la construcción de una estación de ferrocarril en el centro de la ciudad de Terrassa (Fig. 1), muy cerca de donde se descubrió el yacimiento pleistoceno de Cal Guardiola (Postigo Mijarras et al. 2007 y referencias incluidas), sacó a la luz una secuencia de unos $20 \mathrm{~m}$ de potencia, compuesta por sedimentos aluviales asociados al torrente de Vallparadís. Abundantes restos arqueológicos y paleontológicos (Fig. 2) han aparecido en las unidades geológicas diferenciadas (denominadas 1 a 12, de techo a muro). La excavación se centró en el nivel arqueológico más rico (nivel 10), ubicado en la parte superior de la unidad 7. Considerado como el nivel de referencia arqueológica para Vallparadís, contenía una industria lítica arcaica abundante atribuida al Modo 1 u Olduvaiense (Lumley et al. 2009; Martínez et al. 2010). Sus características tecno-tipológicas son similares a las de los conjuntos de otros yacimientos antiguos mediterráneos, como Gran Dolina-TD6, Pont-de-Lavaud, Dmanisi o Bizat Ruhama (Martínez et al. 2010). Esta industria se asociaba con una fauna rica y diversa donde se identificaron 21 taxones de grandes mamíferos (Alba et al. 2008) y algunos de micromamíferos (Martínez et al. 2010). Además se identificó actividad humana por la presencia de marcas de corte sobre algunos restos paleontológicos (Martínez et al. 2010).

Desde el punto de vista de la biocronología, el estudio de la macrofauna ha permitido identificar una asociación típica del epi-Villafranquiense (Martínez et al. 2010). Los taxones villafranquienses (Rook y Martínez-Navarro 2010), como Pseudodama vallonetensis, Equus altidens, Stephanorinus hundsheimensis, Hippopotamus antiquus, Canis mosbachensis, Panthera gombasgoezensis y Pachycrocuta brevirostris presentes

tigraphique et géochronologique des hominidés fossiles du site de Dmanissi en Géorgie. Tesis de doctorado no editada. Muséum National d'Histoire Naturelle. Paris.

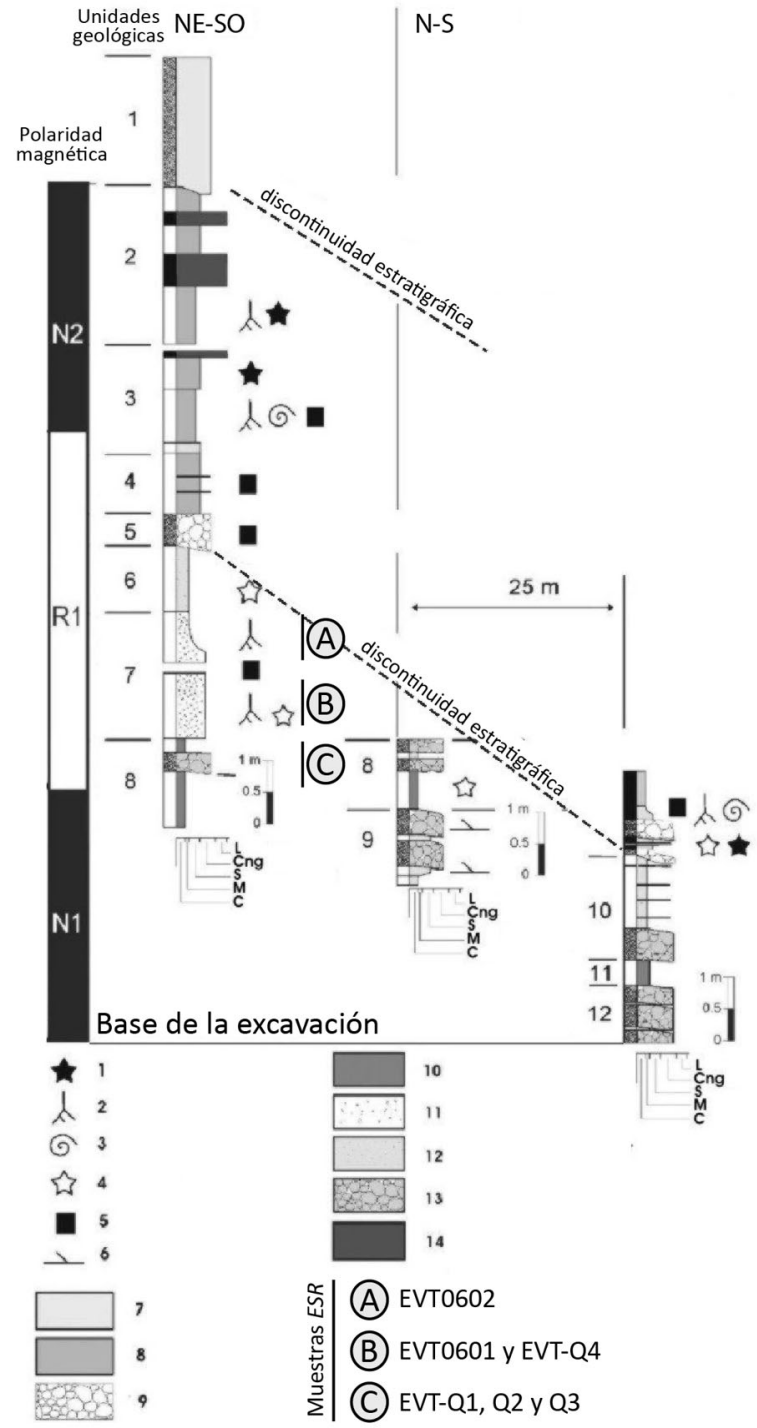

Fig. 2. Yacimiento de Vallparadís (Terrassa, Barcelona): Estratigrafía sintética (Martínez et al. 2010) y posición de las muestras ESR (dientes: EVT0601 y EVT0602; cuarzo: EVT-Q1, EVT-Q2, EVT-Q3 y EVT-Q4). EVT: acrónimo del yacimiento. Símbolos: 1) material orgánico y restos de madera fósil, 2) trazas de raíces, 3) restos de gasterópodos, 4) restos de $\left.\mathrm{CaCO}_{3}, 5\right)$ niveles con industria lítica, 6) laminaciones entrecruzadas, 7) terraza del Pleistoceno superior, 8) arcillas y lodos con gasterópodos, 9) unidad 5, 10) arcillas rojas y lodos, 11) unidad 7, 12) arcillas marrones y lodos, 13) conglomerados, 14) paleo-suelo. $(\mathrm{L}=$ Calizas, $\mathrm{Cng}=$ Conglomerado, $\mathrm{S}=$ Arena, $\mathrm{M}=$ Lodo, $\mathrm{C}=$ Arcilla).

también en los yacimientos españoles de Orce (Martínez-Navarro et al. 2003), Sima del Elefante (Carbonell et al. 2008) y Gran Dolina (Rodríguez et al. 2001) se mezclan con otros más 
característicos del Galeriense, tales como Elephas Antiquus y Ursus deningeri (Palombo y Ferretti 2005; Palombo et al. 2008).

El estudio de la microfauna de la unidad 7, ha identificado la asociación Mimomys savini - Iberomys huescarensis generalmente considerada como un buen marcador cronológico (Agustí et al. 2007). Dicha asociación permite posicionar Vallparadís en la misma biozona que los yacimientos de Huéscar-1 (España, Alberdi et al. 2001), le Vallonnet (Francia), Untermaßfeld (Alemania, Kahlke et al. 2005), o Sima del Elefante, es decir, en una biozona más antigua que la de Atapuerca Gran Dolina-TD6 (Agustí y Madurell 2003; Agustí et al. 2007).

El estudio paleomagnético de toda la secuencia ha revelado tres magnetozonas principales (Fig. 2) de muro a techo (Martínez et al. 2010): la primera tiene polaridad normal (N1, situado en la parte inferior de la unidad 8), la segunda inversa (R1, unidades 8 a 3 ) y la tercera polaridad normal (N2, de la unidad 3 hasta el techo). El nivel arqueológico 10 ubicado en la unidad 7 está posicionado en la magnetozona $\mathrm{R} 1$, indicando entonces una edad anterior al límite Brunhes-Matuyama $(0,78 \mathrm{Ma})$.

\section{EL MÉTODO DE DATACIÓN POR ESR}

La datación por ESR forma parte del grupo de métodos paleodosimétricos con los basados en los fenómenos de la luminiscencia (TL, OSL...). A diferencia de los métodos radiométricos (potasio-argón, uranio-torio (U-Th) (9), radiocarbono...) que evalúan directamente la radiactividad natural, los métodos paleodosimétricos detectan los efectos de dicha radiactividad sobre las muestras geológicas o arqueológicas. En este caso, se mide la energía absorbida por la muestra (dosis total) en función de la cantidad de radiación a la que ha sido sometida durante su historia. Dicha dosis procede de las radiaciones alfa, beta y gamma emitidas por los radionucléidos presentes en la propia muestra (componente interno) y en su entorno más cercano (componente externo), a los cuales se añade un componente procedente de la radiación cósmica. Estas radiaciones ionizantes inducen movimientos en la estructura electrónica de los minerales y algunas cargas eléctricas pue-

(9) Uranium-series (US). den ser atrapadas dentro de los defectos puntuales de la estructura cristalina, formando una entidad llamada "centro paramagnético". Esta entidad genera una señal detectable por espectrometría ESR cuya intensidad es proporcional a la cantidad de cargas atrapadas en la celda cristalina. El método de datación por ESR se basa en la cuantificación de estas cargas atrapadas, cuya cantidad está directamente relacionada con la dosis de radiación absorbida por la muestra y depende tanto de la intensidad de la radiación (tasa de dosis) como de la duración de la exposición a la radiactividad (Grün 1989; Ikeya 1993; Rink 1997).

En la datación por ESR, la muestra es considerada como un dosímetro, es decir, un material capaz de registrar y restituir la dosis absorbida procedente de las diferentes radiaciones ionizantes a las que ha sido sometido. La edad ESR se calcula a partir de la siguiente ecuación:

$$
D_{E}=\int_{0}^{T} D(t) d t
$$

donde $D_{E}$ es la dosis equivalente (expresada en Gray, Gy), $D(t)$ es la tasa de dosis ( $\mu \mathrm{Gy} / \mathrm{a}$ o Gy/ka) y $T$ es la edad de la muestra. En el caso de una tasa de dosis constante en el tiempo, la ecuación se puede simplificar de la siguiente manera:

$$
T=\frac{D_{E}}{D}
$$

En las secciones 3.1 y 3.2 se detallarán las características particulares de la datación de dientes fósiles y granos de cuarzo.

\subsection{Aplicación a los dientes fósiles: el método combinando $E S R$ y $U$-Th}

La principal dificultad de la datación de un diente fósil por ESR es la complejidad del sistema en sí mismo, ya que hay que considerar los diferentes tejidos dentales que lo constituyen (esmalte, dentina y, a veces, cemento, Hillson 2005). En el caso de una geometría, dentina -esmalte- cemento, la ecuación es la siguiente:

$$
\begin{gathered}
D_{E}=\int_{0}^{T}\left[D_{\text {esmalte }}(t)+D_{\text {dentina }}(t)+D_{\text {cemento }}(t)+\right. \\
\left.+D_{\text {se dimento }}(t)+D_{\text {cósmi cos }}(t)\right] d t
\end{gathered}
$$


La dosis interna corresponde a la producida dentro del esmalte y la dosis externa a los componentes asociados a los otros tejidos dentales (dentina y cemento), el sedimento y los rayos cósmicos. La particularidad de la datación de dientes fósiles reside en estos componentes ligados a los tejidos dentales, puesto que éstos difieren a nivel de mineralización, de composición química (Driessens 1980; Hillson 2005) y de sensibilidad a los procesos diagenéticos (Piepenbrink 1989; Kohn et al. 1999; Dauphin y Williams 2004), en particular al proceso de incorporación de uranio. Los tejidos dentales funcionan como sistemas abiertos frente a los elementos de la serie radiactiva de desintegración del ${ }^{238} \mathrm{U}$, lo que significa que el contenido en uranio del tejido dental varía con el tiempo. Por tanto, es indispensable tener en cuenta este parámetro en la determinación de la dosis anual. Para describir su evolución, Grün et al. (1988) sugieren la combinación de los métodos ESR y U-Th (ESR-US). Este enfoque se basa en la modelización matemática de la cinética de incorporación del uranio en los tejidos dentales, descrita a través de un parámetro específico $p$. Este modelo llamado US (U-series) utiliza la siguiente ecuación:

$$
U(t)=U_{m}\left[\frac{t}{T}\right]^{p+1}
$$

donde $U_{m}$ es la concentración de uranio medida hoy, $T$ la edad de la muestra y $U(t)$ la concentración de uranio en el tiempo $t$. El parámetro $p$ es siempre superior a -1 (Fig. 3). Básicamente, se utilizan las medidas de los isótopos de la serie del uranio para relacionar $p$ y las edades $U-T h$ para cada tejido dental. Estas relaciones y el resto de parámetros de tasa de dosis determinan la evolución de la dosis total en función de la edad. La proyección de la $D_{E}$ medida sobre esta función da una edad ESR-US combinada y dicha edad permite obtener el valor de $p$ para cada tejido (Grün et al. 1988; Grün 2007).

Antes de la aplicación del modelo US, los modelos convencionales normalmente utilizados para calcular una edad ESR consideraban un tipo predeterminado de incorporación. El modelo de "incorporación temprana" (Early Uptake, EU) se basa en la idea de una rápida acumulación de uranio en la muestra poco después de su enterramiento, en una aproximación de sistema cerrado
(Szabo 1979). En el modelo de "incorporación lineal" (Linear Uptake, $L U$ ), sin embargo, ésta es una incorporación constante en el tiempo (Ikeya 1982). Finalmente, el modelo de "incorporación reciente" (Recent Uptake, RU), supone una incorporación tardía de dicho elemento (Blackwell 1992). Clásicamente, se asumía que uno de estos modelos podía corresponder a las muestras de un yacimiento dado o bien que la edad verdadera estaba entre las edades ESR-EU y ESR-LU (Grün y Stringer 1991). Sin embargo, esto no es satisfactorio, porque estos modelos inducen incertidumbres largas si la concentración de uranio en los tejidos dentales es alta. En otras palabras, cuanto más alta es la concentración de uranio, mayor es la diferencia entre las edades $E S R-E U, E S R-L U$ y $E S R-R U$ (Grün y McDermott 1994). Además, como ha indicado Grün (2009a), muchas veces la mejor estimación de edad está fuera del rango $E U-L U$, especialmente para las muestras procedentes de yacimientos al aire libre. El modelo US permite entonces solucionar este problema de incorporación desconocida. Los resultados obtenidos en Gran Dolina por Falguères et al. (1999) muestran que este método ESR-US combinado puede ser aplicado de modo rutinario hasta los 800 ka. Falguères et al. (2010) concluyen que dicho método es especialmente adecuado para muestras del Pleistoceno medio.

El modelo US tiene varias ventajas con respecto a los modelos convencionales. En primer lugar, la determinación del factor $p$ permite carac-

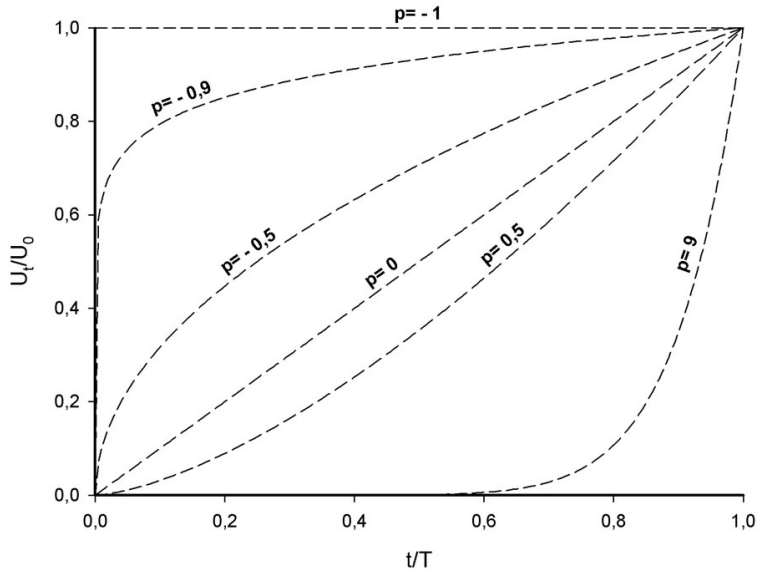

Fig. 3. Forma de incorporación del uranio en función del valor del parámetro $p$ seleccionado (Grün et al. 1988). Casos especiales: $p=-1$ para el modelo Early Uptake (EU), $p=0$ para el modelo Linear Uptake $(L U)$ y $p=1$ ó 10 para el Recent Uptake (RU).

T. P., 68, N. ${ }^{\circ} 1$, enero-junio 2011, pp. 7-24, ISSN: 0082-5638 doi: $10.3989 /$ tp.2011.11056 
terizar la cinética de incorporación del uranio dentro de los tejidos dentales: incorporación temprana si $-1<p<0$ o incorporación tardía si $p>0$ (Fig. 3). Además los modelos convencionales pasan a ser casos especiales del modelo US, correspondientes a valores precisos de $p(p=-1$ para el modelo $E U, p=0$ para el modelo $L U$ y $p=1$ ó 10 para el modelo $R U$ ). El método ESR-US combinado también permite determinar diferentes incorporaciones para los distintos tejidos de un mismo diente, mediante el cálculo de un $p$ específico para cada uno (Grün, 2009a). Por último, el modelo US no asume a priori la forma de incorporación del uranio, sino que determina matemáticamente a partir de los datos isotópicos medidos en los tejidos dentales (concentración en uranio, ${ }^{234} \mathrm{U} /{ }^{238} \mathrm{U},{ }^{230} \mathrm{Th} /{ }^{234} \mathrm{U}$ ). La consecuencia es que sólo existe una posible edad ESR-US combinada para un conjunto de datos medidos (datos isotópicos de los tejidos y del sedimento, $D_{E}$ calculada, contenido de agua...), siempre, y cuando sea posible calcular la edad.

\subsection{Aplicación a los granos de cuarzo extraídos de sedimentos}

La datación está basada en el mismo principio que la OSL: la puesta a cero de la señal paleodosimétrica bajo la influencia de la luz solar, fenómeno conocido como blanqueo óptico. A partir del momento en el que el cuarzo es enterrado y pierde el contacto con la luz solar, la señal ESR aumenta como consecuencia de la radiactividad natural (detalles en Voinchet et al. 2004). El evento datado es, por tanto, el entierro de la muestra.

El cuarzo posee varios centros paramagnéticos potencialmente útiles con fines geocronológicos (Marfunin 1979; Weil 1984; Ikeya 1993): centro Germanio (Ge), centro Titanio (Ti), centro Alu- minio (Al), centro E', centro OHC... cuyas características propias (en términos de sensibilidad a la radiación, estabilidad térmica, cinética de blanqueo...) los hacen más o menos atractivos en función del contexto geológico y del período de tiempo que se quiere datar (Ikeya 1993).

El centro Al es probablemente el más utilizado en geocronología y tiene dos principales ventajas. Primero, como el aluminio es el elemento traza más abundante en el cuarzo (su concentración puede ser de varios miles de ppm, ver ejemplos en Preusser et al. 2009), la señal ESR del centro Al se observa siempre, algo que no ocurre con las asociadas a otros centros (Yokoyama et al. 1985; Duttine et al. 2002). Además, siendo mucho menos radio-sensible que el centro Ti por ejemplo, el centro Al no muestra saturación a dosis de irradiación muy altas (>60 kGy, véase Lin et al. 2006). Con una duración de vida promedia estimada a 7,4*109 años (Toyoda y Ikeya 1991) este centro puede, potencialmente, ser utilizado para datar períodos muy antiguos, anteriores al inicio del Cuaternario (un ejemplo en Laurent et al. 1998).

\section{MUESTREO ESR}

En 2006, dos dientes de équidos fueron seleccionados entre los ejemplares de las colecciones del yacimiento (Tab. 1). La primera muestra (EVT0601) procede del cuadro U-30 (Fig. 4) y se localiza estratigráficamente en el nivel arqueológico 10 de la unidad 7 (Fig. 2). La segunda muestra (EVT0602) fue recogida en el cuadro P-26 y también se localiza en la unidad 7, pero en el contacto entre los niveles arqueológicos 9 y 10. Se colocaron dos dosímetros $T L$ en la secuencia estratigráfica, manteniéndose durante 10 meses. El primero fue situado en la posición exacta en la que se encontró el diente EVT0601 (sección del cuadro P-26). El segundo fue insta-

\begin{tabular}{|c|c|c|c|c|c|c|}
\hline \multicolumn{1}{|c|}{ Muestra } & Tipo & $\begin{array}{c}\text { Referencia } \\
\text { excavación }\end{array}$ & Cuadrícula & $\mathbf{N .}^{{ }^{\circ}}$ & $\begin{array}{c}\text { Profundidad } \\
\text { (cm) }\end{array}$ & $\begin{array}{c}\text { Unidad } \\
\text { geológica (nivel } \\
\text { arqueológico) }\end{array}$ \\
\hline EVT0601 & Equus sp. & EVT503 & U-30 & - & 424 & $7(10)$ \\
EVT0602 & Equus sp. & EVT2755 & P-26 & 43 & 383 & $7(9 / 10)$ \\
\hline
\end{tabular}

Tab. 1. Yacimiento de Vallparadís (Terrassa, Barcelona): inventario de los dientes datados por el método ESR-US combinado.

T. P., 68, N. ${ }^{\circ}$ 1, enero-junio 2011, pp. 7-24, ISSN: 0082-5638

doi: $10.3989 /$ tp. 2011.11056 
lado en el contacto entre los niveles 9 y 10 correspondiente a la localización estratigráfica del diente EVT0602.
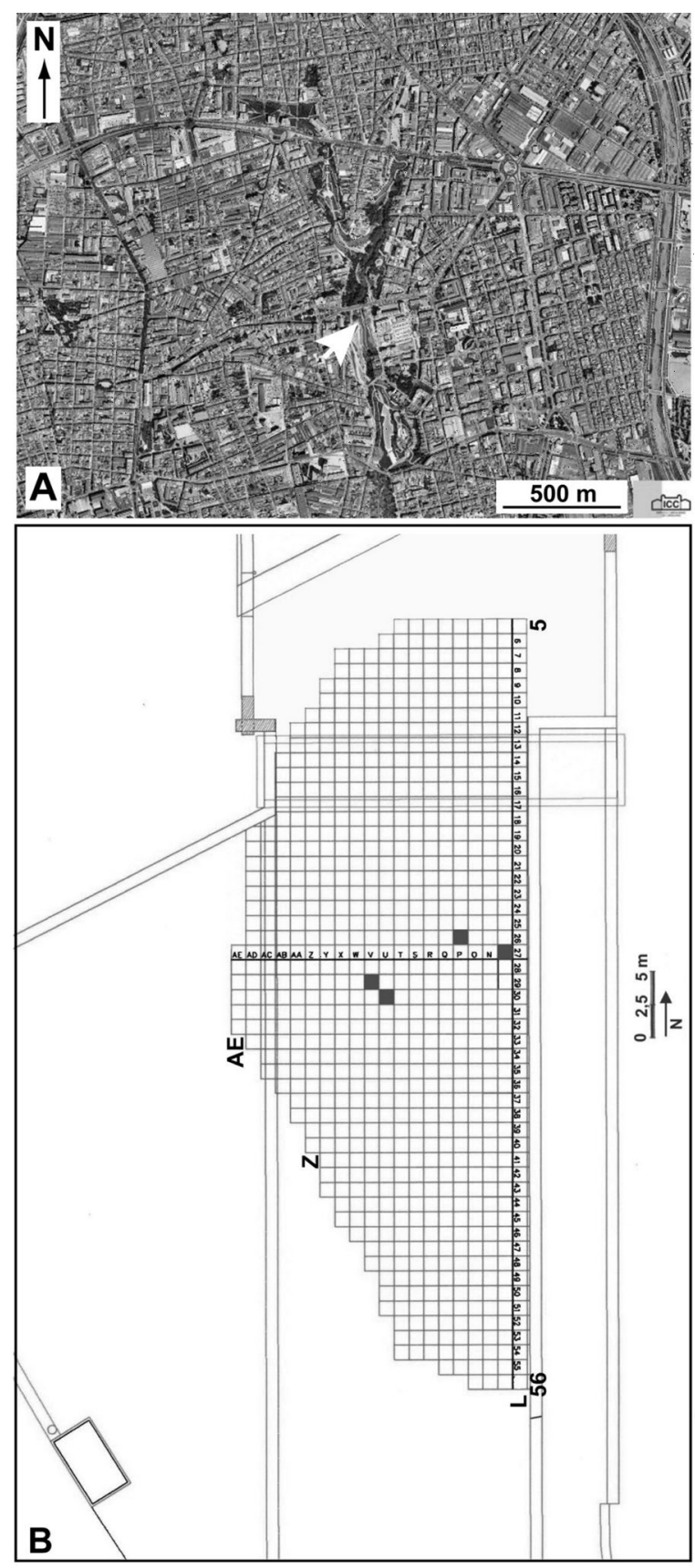

Fig. 4. A: Localización de la zona de excavación (flecha blanca) dentro del parque de Vallparadís y de la ciudad de Terrassa. B: Localización espacial de las muestras ESR (cuadrículas llenas) al interior de la zona de excavación (altitud 275 m.s.n.m.). Muestra (cuadrícula): EVT0601 (U-30), EVT0602 (P-26), EVT-Q1 (V-29), EVT-Q2 (V-29), EVT-Q3 (V-29), EVT-Q4 (M-27).
Además, se tomaron in situ 4 muestras de sedimentos (EVT-Q1 a Q4) para datar los granos de cuarzo. Las muestras EVT-Q1 a Q3 proceden de la sección del cuadro V-29 y se localizan en la unidad geológica 8 (Fig. 2), en los niveles inferiores al nivel arqueológico 10. La muestra Q4 fue recogida en el cuadro M-27 (Fig. 4), en una capa de depósitos groseros de la base del nivel arqueológico 10 (unidad 7). No se hizo ninguna dosimetría gama in situ para estas muestras. Sin embargo, con el objetivo de considerar la posible heterogeneidad del ambiente sedimentario en términos de radiactividad, se tomaron varias muestras de sedimento alrededor de los puntos de muestreo destinados a la datación.

\section{PROTOCOLO ANALÍTICO}

\subsection{Datación de dientes fósiles}

Los diferentes tejidos (cemento, esmalte y dentina) fueron extraídos de la cara vestibular de cada diente y separados mecánicamente. Los análisis isotópicos fueron realizados por espectrometría alfa (Bischoff et al. 1988), y por espectrometría gamma (Yokoyama y Nguyen 1980) para obtener datos de los elementos de las series del uranio. Dichos datos se encuentran en la tabla 2.

Una parte del esmalte fue molida y tamizada, una vez que las superficies interna y externa fueron exhaustivamente limpiadas para eliminar el efecto de la radiación alfa exterior. A continuación, se recuperó la fracción granulométrica de 100-200 $\mu \mathrm{m}$, que se dividió en varias alícuotas. Dichas alícuotas fueron irradiadas en el Laboratoire National Henri Becquerel (LNE-LNHB, Francia) con una fuente gamma calibrada de ${ }^{60} \mathrm{Co}$, utilizando 15 dosis de irradiación con una distribución exponencial (Grün y Rhodes 1991): $0,100,160,250,400,630,1000,1600,2200$, $3600,5600,8900,12600,16000$ y $20000 \mathrm{~Gy}$.

Las medidas ESR fueron realizadas en el $\mathrm{Mu}$ séum National d'Histoire Naturelle (Francia) con un espectrómetro EMX Bruker (banda X) a temperatura ambiente, con los parámetros de adquisición indicados en la tabla 3 . Cada conjunto de alícuotas fue medido 3 veces, con varios días de diferencia entre cada medida, para comprobar la reproductibilidad de los datos. A continuación, se midió la intensidad ESR correspondiente a la 


\begin{tabular}{|c|c|c|c|c|c|c|c|}
\hline Muestra & T. & $\mathbf{U}(\mathbf{p p m})$ & ${ }^{234} \mathbf{U} /{ }^{238} \mathbf{U}$ & ${ }^{230} \mathbf{T h} /{ }^{234} \mathbf{U}$ & ${ }^{222} \mathbf{R n} /{ }^{230} \mathbf{T h}$ & E. esm. ${ }^{*}(\mu \mathbf{m})$ & E. elim.** $(\mu \mathbf{m})$ \\
\hline & $\mathrm{C}$ & $27,400 \pm 0,082$ & $2,102 \pm 0,050$ & $1,084 \pm 0,036$ & $0,21 \pm 0,05$ & & $(2) 80 \pm 10$ \\
EVT0601 & $\mathrm{D}$ & $105,95 \pm 1,980$ & $2,030 \pm 0,026$ & $0,991 \pm 0,023$ & $0,44 \pm 0,10$ & & $(1) 90 \pm 11$ \\
& $\mathrm{E}$ & $2,350 \pm 0,070$ & $1,801 \pm 0,039$ & $0,997 \pm 0,032$ & $1,00 \pm 0,05$ & $1440 \pm 180$ & \\
& & & & & & $(2) 40 \pm 5$ \\
EVT0602 & $\mathrm{C}$ & $36,220 \pm 0,850$ & $2,100 \pm 0,035$ & $1,191 \pm 0,035$ & $0,18 \pm 0,05$ & & $(1) 60 \pm 8$ \\
& $\mathrm{D}$ & $90,850 \pm 1,750$ & $2,100 \pm 0,027$ & $1,115 \pm 0,029$ & $0,30 \pm 0,10$ & & \\
& $\mathrm{E}$ & $4,150 \pm 0,120$ & $1,752 \pm 0,034$ & $1,051 \pm 0,036$ & $0,83 \pm 0,08$ & $1510 \pm 189$ & \\
\hline
\end{tabular}

Tab. 2. Yacimiento de Vallparadís (Terrassa, Barcelona): datos isotópicos medidos en los dientes de équidos (Martínez et al. 2010). Los errores estadísticos asociados son de $1 \sigma$. T.: tejido dental, C: cemento, D: dentina y E: esmalte. (*) espesor (E.) inicial del esmalte (esm.); (**) espesor de esmalte suprimido para el cálculo de la edad ESR-US, (1): parte interna del esmalte eliminada (elim.) (lado de la dentina); (2): parte externa del esmalte eliminada (lado del cemento).

\begin{tabular}{|l|c|c|}
\hline \multicolumn{1}{|c|}{ Parámetros } & Esmalte & Cuarzo \\
\hline $\begin{array}{l}\text { Campo magnético } \\
\text { Anchura de barrido }\end{array}$ & & \\
Tiempo de barrido & $12 \mathrm{mT}$ & $9 \mathrm{mT}$ \\
Resolución & $21 \mathrm{~s}$ & $42 \mathrm{~s}$ \\
& 1024 puntos & 1024 puntos \\
\hline Micro-onda & & \\
Frecuencia & $9,8-9,9 \mathrm{GHz}$ & \\
Potencia & $1 \mathrm{~mW}$ & $5 \mathrm{~mW}$ \\
\hline Señal & & \\
Frecuencia de modulación & $100 \mathrm{kHz}$ & $100 \mathrm{kHz}$ \\
Amplitud de modulación & $0,1 \mathrm{mT}$ & $0,1 \mathrm{mT}$ \\
Tiempo de conversión & $20 \mathrm{~ms}$ & $40 \mathrm{~ms}$ \\
Constante de tiempo & $5 \mathrm{~ms}$ & $40 \mathrm{~ms}$ \\
Número de barridos & 1 & 1 \\
\hline
\end{tabular}

Tab. 3. Parámetros de adquisición de las medidas por Resonancia Paramagnética Electrónica (ESR) utilizados para las muestras de esmalte y de cuarzo del yacimiento de Vallparadís (Terrassa, Barcelona).

amplitud entre los picos T1 y B2 de la señal ESR del esmalte (Fig. 5) (Grün 2000). Las dosis equivalentes $\left(D_{E}\right)$ y errores asociados fueron calculados con un programa GW-Basic no-comercial (Yokoyama et al. 1985), ajustando una función exponencial de saturación sencilla (10) a través de los puntos experimentales. No se consideró ninguna ponderación de los puntos experimentales durante el ajuste, pero se restringió la exactitud sobre la $D_{E}$ con la alícuota natural. La figura 6 muestra las curvas de crecimiento ESR obtenidas.

Las edades ESR-US combinadas fueron calculadas con el programa DATA (Grün 2009b), utili-

(10) Single Saturating Exponential (SSE). zando la misma geometría de muestra para los dos dientes: dentina/esmalte/cemento. Se utilizó una eficacia alfa de 0,13 $\pm 0,02$ (Grün y Katzenberger-Apel 1994) y factores de atenuación beta
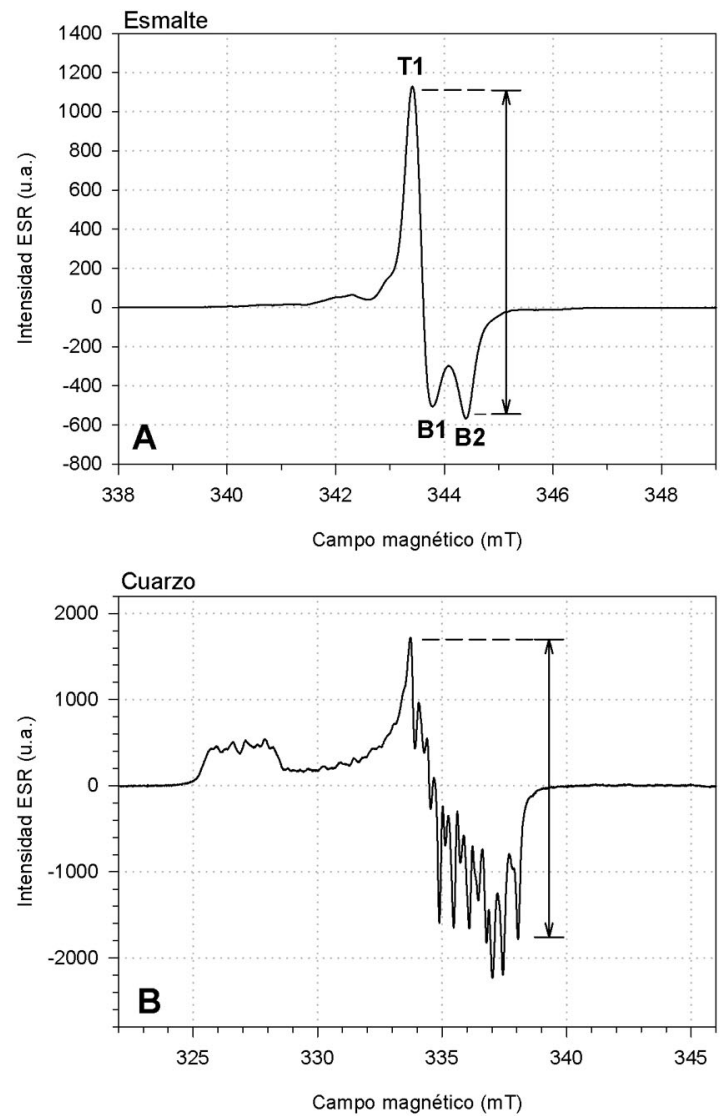

Fig. 5. Ejemplos de señales ESR. A: de esmalte dental. Se extrae la intensidad ESR de las amplitudes entre los picos T1 y B2. B: del centro Al presente en el cuarzo. Se mide la intensidad ESR de la señal Al entre el techo del primer pico y la base del último. 

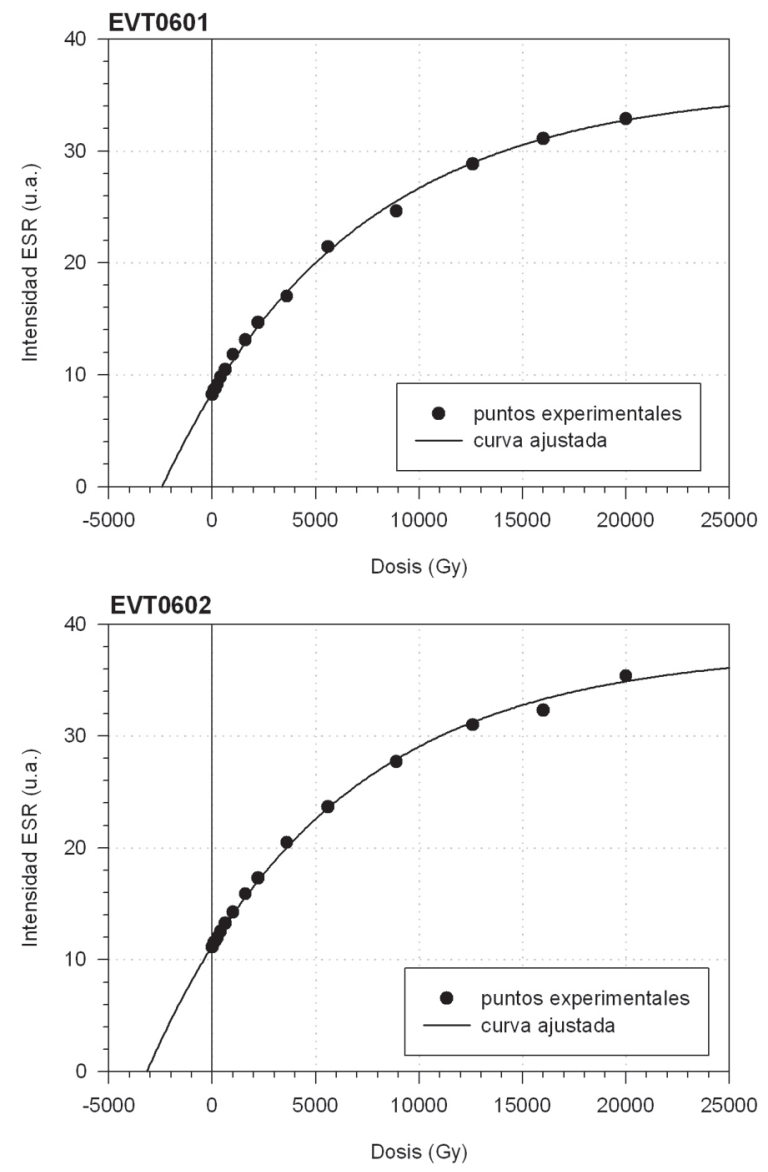

Fig. 6. Curva de crecimiento ESR de los dientes de équidos EVT0601 y EVT0602 para los cuales se ajustó una función de tipo exponencial (SSE).

por Monte-Carlo (11) basados en el espesor del esmalte dental y de las capas externas eliminadas. Se estimó el contenido en agua a $3 \pm 1 \%$ en el esmalte, a $5 \pm 3 \%$ en la dentina y el cemento y a 15 $\pm 5 \%$ en el sedimento, este último basado en la diferencia de peso antes y después de una estancia de un par de semanas en la estufa. El efecto de las pérdidas de $\mathrm{Ra}$ y $\mathrm{Rn}$ en cada tejido fue determinado por combinación de datos de espectrometría alfa y gama (Bahain et al. 1992). Se usó la espectrometría de rayos gamma para determinar el contenido en radioelementos (U, Th y K) del sedimento tomado in situ. Los factores de Adamiec y Aitken (1998) fueron utilizados para las con-

(11) Marsh, R. 1999: Beta-gradient isochrons using electron paramagnetic resonance: towards a new dating method in archaeology. Tesis de doctorado no editada. McMaster University, Hamilton, Ont., Canada. versiones en tasa de dosis. Se midieron las tasas de dosis gamma in situ con dosímetros TL $\left(\mathrm{CaSO}_{4}\right.$ : Dy). El componente cósmico fue calculado con los datos de Prescott y Hutton (1988, 1994). El error asociado a la edad corresponde a la suma cuadrática de los errores asociados a la tasa de dosis y $\mathrm{D}_{\mathrm{E}}$. Todos los datos vinculados con el cálculo de edades ESR-US se encuentran en la tabla 4.

\subsection{Datación de granos de cuarzo}

Las muestras de sedimento fueron preparadas según el protocolo descrito en Voinchet et al. (2007). Primero fueron tamizadas para recuperar la fracción granulométrica de 100-200 $\mu \mathrm{m}$ para luego ser sometidas a un múltiple ataque químico: $\mathrm{HCl} 6 \mathrm{~N}$ para disolver los carbonatos, $\mathrm{H}_{2} \mathrm{O}_{2}$ para quitar la materia orgánica y $\mathrm{HF}(40 \%)$ durante 30 minutos para eliminar los feldespatos y la superficie exterior de los granos de cuarzo. Para finalizar, se separaron minerales pesados y ligeros con bromoformo.

Cada muestra fue dividida en diferentes alícuotas que, posteriormente, serían irradiadas con una fuente de rayos gamma panorámica de ${ }^{60} \mathrm{Co}$ en el Commissariat à l'Energie Atomique (Saclay, Francia) (Dolo et al. 1996). Las dosis solici-

\begin{tabular}{|c|c|c|c|}
\hline \multicolumn{2}{|c|}{ Muestra } & EVT0601 & EVT0602 \\
\hline \multicolumn{2}{|c|}{ Profundidad (m) } & $9 \pm 2$ & $9 \pm 2$ \\
\hline \multirow{5}{*}{\multicolumn{2}{|c|}{$\begin{array}{l}\alpha+\beta \text { interno }(\mu \mathrm{Gy} / \mathrm{a}) \\
\beta \text { dentina }(\mu \mathrm{Gy} / \mathrm{a}) \\
\beta \text { cemento }(\mu \mathrm{Gy} / \mathrm{a}) \\
\gamma \text { sedimentos }+ \text { cósmico }(\mu \mathrm{Gy} / \mathrm{a}) \\
\text { Tasa de dosis total }(\mu \mathrm{Gy} / \mathrm{a})\end{array}$}} & $1045 \pm 56$ & $2935 \pm 370^{*}$ \\
\hline & & $676 \pm 97$ & $1313 \pm 130^{*}$ \\
\hline & & $182 \pm 26$ & $554 \pm 55^{*}$ \\
\hline & & $1041 \pm 56$ & $1042 \pm 55$ \\
\hline & & $2944 \pm 244$ & $5844 \pm 400^{*}$ \\
\hline \multicolumn{2}{|l|}{$\mathrm{D}_{\mathrm{E}}(\mathrm{Gy})$} & $2432 \pm 65$ & $3151 \pm 69$ \\
\hline Factor $p$ & $\begin{array}{l}\text { esmalte } \\
\text { dentina } \\
\text { cemento }\end{array}$ & $\begin{array}{l}-0,54 \\
-0,47 \\
-0,61\end{array}$ & $\begin{array}{l}-1 \\
-1 \\
-1\end{array}$ \\
\hline \multicolumn{2}{|c|}{ Edad ESR-US (Ma) } & $0,83 \pm 0,07$ & $0,54 \pm 0,04^{(1)}$ \\
\hline
\end{tabular}

Tab. 4. Yacimiento de Vallparadís (Terrassa, Barcelona): edades ESR-US combinadas y datos asociados obtenidos para los dientes fósiles (adaptado de Martínez et al. 2010). (*): valores calculados considerando un modelo de incorporación Early Uptake (EU) para todos los tejidos dentales $(p=-1)(1)$. La edad de EVT0602 corresponde a una edad ESR-EU, calculada mediante el programa DATA de Grün (2009b).

T. P., 68, N. ${ }^{\circ} 1$, enero-junio 2011, pp. 7-24, ISSN: 0082-5638 doi: $10.3989 /$ tp.2011.11056 


\begin{tabular}{|c|c|c|c|c|c|c|c|}
\hline \multirow{2}{*}{ Muestra } & \multirow{2}{*}{$\mathbf{C}_{\mathbf{B}}$ (\%) } & \multicolumn{3}{|c|}{ EXP+LIN } & \multicolumn{3}{c|}{ SSE } \\
\cline { 3 - 8 } & & $\mathbf{D}_{\mathbf{E}}$ (Gy) & $\mathbf{X}^{\mathbf{2}}$ & $\mathbf{R}^{\mathbf{2}}$ & $\mathbf{D}_{\mathbf{E}}$ (Gy) & $\mathbf{X}^{\mathbf{2}}$ & $\mathbf{R}^{\mathbf{2}}$ \\
\hline \multirow{2}{*}{ EVT-Q1 } & 60 & $1894 \pm 201$ & 0,00049 & 0,99783 & $2697 \pm 319$ & 0,00240 & 0,98795 \\
EVT-Q2 & 59 & $2044 \pm 418$ & 0,00244 & 0,98772 & $2175 \pm 312$ & 0,00350 & 0,98054 \\
EVT-Q3 & 56 & $2132 \pm 701$ & 0,00256 & 0,98516 & $3856 \pm 633$ & 0,00351 & 0,97671 \\
EVT-Q4 & 57 & $1286 \pm 365$ & 0,00314 & 0,98493 & $2818 \pm 517$ & 0,00546 & 0,97007 \\
\hline
\end{tabular}

Tab. 5. Yacimiento de Vallparadís (Terrassa, Barcelona): datos ESR asociados con el ajuste de las funciones exponencial + lineal $(E X P+L I N)$ y exponencial $(S S E)$ a los puntos experimentales de las muestras de cuarzo EVT-Q1 a Q4. $\mathrm{C}_{\mathrm{B}}=$ componente blanqueada; $\mathrm{X}^{2}=$ chi cuadrado; $\mathrm{R}^{2}=$ coeficiente de determinación.

tadas fueron las siguientes (tasa de dosis de 2000 $\mathrm{Gy} / \mathrm{h}$ ): 400, 630, 1000, 1600, 2500, 4000, 6300, 10000,16000 y 25000 Gy. Durante la irradiación se colocaron diferentes dosímetros de alanina entre las muestras con el objetivo de controlar la dosis de irradiación realmente recibida por cada muestra (12). Mientras tanto, la componente residual (no-blanqueable) de la señal ESR asociada al centro $\mathrm{Al}(E S R-A l)$ fue determinada tras la exposición de una alícuota natural de cada muestra a la luz de un simulador de tipo SOL2 (Hönle) durante $1500 \mathrm{~h}$. El componente blanqueado (expresado en \%) corresponde a la diferencia relativa entre la intensidad ESR del punto natural y la del residual.

Las medidas ESR fueron realizadas en el $\mathrm{Mu}$ séum National d'Histoire Naturelle (Francia) a baja temperatura (alrededor de $110 \mathrm{~K}$ ), con los parámetros de adquisición indicados en la tabla 3. Se midió cada alícuota cada $120^{\circ}$ de rotación en la cavidad y cada serie, a su vez, entre 2 y 4 veces. La intensidad de la señal $E S R-A l$ es determinada midiendo la amplitud entre la parte superior del primer pico $(\mathrm{g}=2,0185)$ y la parte inferior del decimosexto pico $(\mathrm{g}=1,9928)$ (Toyoda y Falguères 2003) (Fig. 5). Los valores de $D_{E}$ y los errores asociados fueron calculados con el programa $\mathrm{Mi}$ crocal Origin utilizando el algoritmo de Levenberg-Marquardt por minimización de chi-cuadrado. Los datos fueron ponderados por $1 / \mathrm{I}^{2}$. El valor de $D_{E}$ fue calculado utilizando dos funciones ajustadas a través de los puntos experimentales hasta el nivel residual: una función $S S E$ y otra función compuesta por la combinación de un término exponencial de saturación sencilla y de un término lineal $(E X P+L I N$; más detalles sobre esta función en Berger 1990; Duval et al. 2009). Las curvas de crecimiento ESR de cada muestra están

(12) Véase nota 6. presentadas en la figura 7. La tabla 5 muestra algunos datos numéricos relacionados con estos procesos de ajuste.

Las componentes alfa, beta y gamma de la tasa de dosis total ( $\left.D^{\prime}\right)$ y sus incertidumbres se calculan mediante las concentraciones en ${ }^{238} \mathrm{U}$, ${ }^{222} \mathrm{Rn},{ }^{232} \mathrm{Th}$ y ${ }^{40} \mathrm{~K}$ del sedimento determinadas por espectrometría gamma (Yokoyama y Van Nguyen 1980). Se utilizaron los factores de conversión en dosis de Adamiec y Aitken (1998). La tasa de dosis interna se considera insignificante, debido a la reducida concentración de radionucléidos que se ha registrado normalmente en los granos de cuarzo (Murray y Roberts 1997; Vandenberghe et al. 2008). Se midió el desequilibrio en la cadena de desintegración del ${ }^{238} \mathrm{U}$ a través de la actividad del ${ }^{222} \mathrm{Rn}$. En este trabajo, se ha utilizado una eficiencia alfa de 0,2 $\pm 0,1$ (Yokoyama et al. 1985), los valores de atenuación alfa y beta para granos esféricos de Brennan et al. (1991) y Brennan (2003) y la atenuación del agua según Aitken (1985). Se ha considerado un contenido de humedad del $15 \pm 5 \%$ para todas las muestras después de haber medido sus respectivos pesos, antes y después, de haberlas introducido durante 3 semanas en una estufa. La tasa de dosis cósmica se calcula mediante la fórmula de Prescott y Hutton (1994), aplicando las correcciones correspondientes a la profundidad, altitud y latitud, con un error asociado de $5 \%$ (Prescott y Hutton 1988). Se ha considerado una profundidad de $11 \pm 2 \mathrm{~m}$ para las muestras Q1 a Q3 y de $9 \pm 2$ m para la muestra Q4. Las componentes beta y gamma fueron calculadas a partir del contenido medio en radionucléidos de cada muestra en sí misma y de las diferentes muestras de sedimento que se tomaron a su alrededor. Los errores asociados a las dosis corresponden a $1 \sigma$, aunque sea una combinación de incertidumbres medida y asumida. Por último, se calculó una 

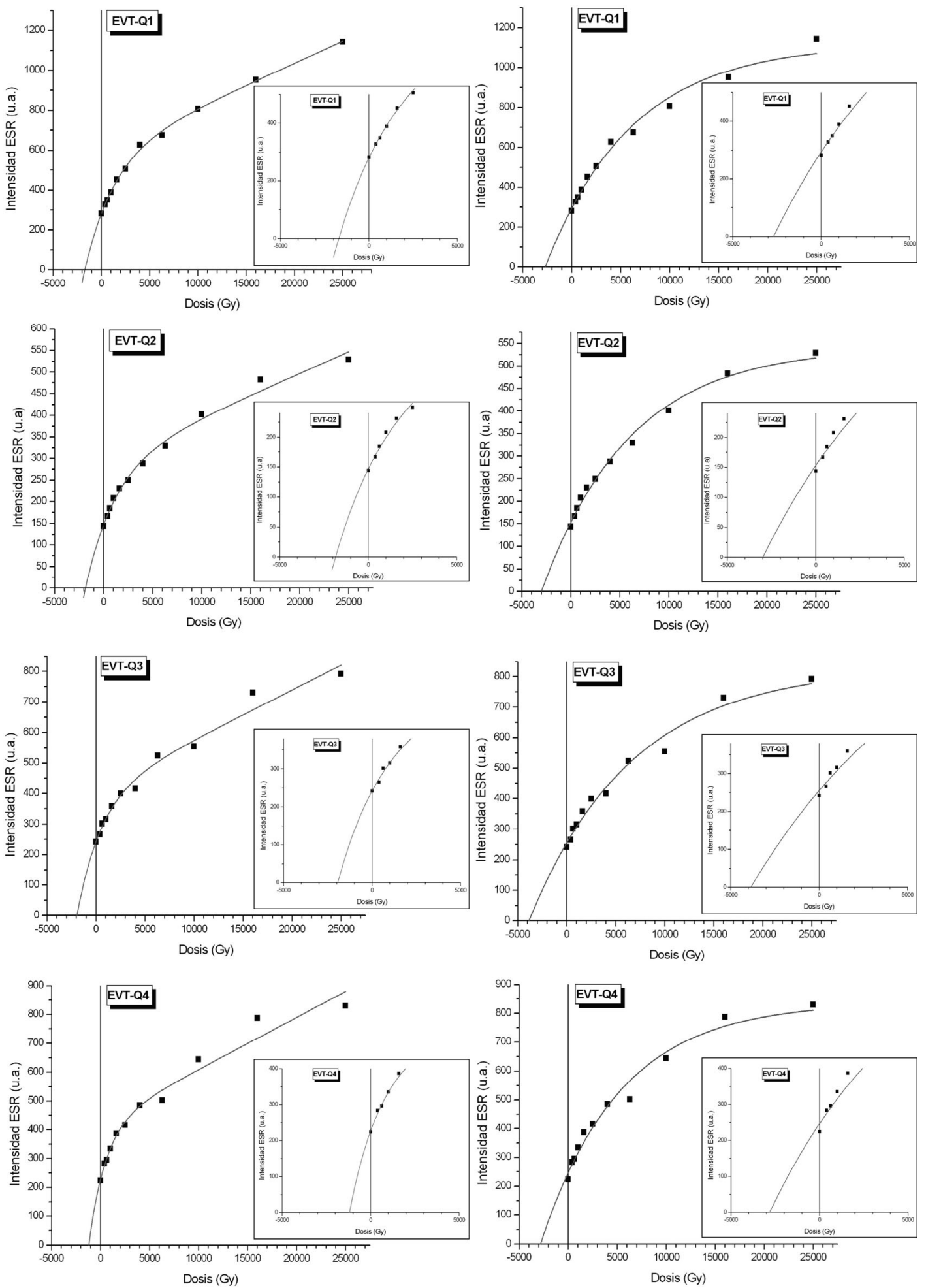

Fig. 7. Curvas de crecimiento ESR obtenidas para las muestras de cuarzo EVT-Q1 a Q4 con el ajuste de dos tipos de funciones: exponencial + lineal ( $E X P+L I N$, columna izquierda) y exponencial (SSE, columna derecha). Encuadrados: ampliación sobre los puntos experimentales correspondiendo a las dosis de irradiación débiles. 


\begin{tabular}{|l|c|c|c|c|}
\hline \multicolumn{1}{|c|}{ Muestra } & EVT-Q1 & EVT-Q2 & EVT-Q3 & EVT-Q4 \\
\hline Unidad (nivel) & $8(12)$ & $8(12)$ & $8(12)$ & $7(10)$ \\
$U(\mathrm{ppm})$ & $1,74 \pm 0,09$ & $2,04 \pm 0,09$ & $1,77 \pm 0,11$ & $1,21 \pm 0,13$ \\
$T h(\mathrm{ppm})$ & $7,82 \pm 0,14$ & $8,52 \pm 0,14$ & $8,74 \pm 0,18$ & $4,23 \pm 0,19$ \\
$K(\%)$ & $1,99 \pm 0,02$ & $1,99 \pm 0,02$ & $2,08 \pm 0,02$ & $1,09 \pm 0,02$ \\
Profundidad $(\mathrm{m})$ & $11 \pm 2$ & $11 \pm 2$ & $11 \pm 2$ & $9 \pm 2$ \\
$D \alpha(\mu \mathrm{Gy} / \mathrm{a})$ & 51 & 57 & 57 & 31 \\
$D_{\beta}(\mu \mathrm{Gy} / \mathrm{a})$ & 1488 & 1530 & 1573 & 845 \\
$D \gamma(\mu \mathrm{Gy} / \mathrm{a})$ & 881 & 984 & 899 & 619 \\
$D_{\cos (\mu \mathrm{Gy} / \mathrm{a})}$ & 47 & 47 & 47 & 59 \\
$D^{\prime}(\mu \mathrm{Gy} / \mathrm{a})$ & $2468 \pm 108$ & $2619 \pm 104$ & $2576 \pm 96$ & $1554 \pm 92$ \\
$D_{E}(\mathrm{~Gy})$ & $1894 \pm 201$ & $2044 \pm 418$ & $0,83 \pm 0,27$ & $1286 \pm 365$ \\
Edad (Ma) & $0,77 \pm 0,23$ & $0,78 \pm 0,16$ & $0,83 \pm 0,24$ \\
\hline
\end{tabular}

Tab. 6. Yacimiento de Vallparadís (Terrassa, Barcelona): datos radiométricos, dosimétricos y cronológicos asociados a las muestras de cuarzo datadas por Resonancia Paramagnética Electrónica (ESR). Los diferentes componentes de la dosis total $D$ ' son presentados: alfa $\left(D_{\alpha}\right)$, beta $\left(D_{\beta}\right)$, gamma $\left(D_{\gamma}\right)$ y cósmico $\left(D_{\text {cos }}\right)$.

edad ESR dividiendo $D_{E}$ por $D^{\prime}$. La tabla 6 presenta los datos radiométricos, dosimétricos y cronológicos.

\section{RESULTADOS Y DISCUSIÓN}

Las curvas de crecimiento ESR de los 2 dientes se ajustan bastante bien a los puntos experimentales (Fig. 6). Las $D_{E}$ son bastante altas, con $2432 \pm 65$ Gy para EVT0601 y $3151 \pm 69$ Gy para EVT0602 (Tab. 4), valores que son más de 2 veces superiores a los obtenidos para las muestras de Gran Dolina-TD6 (Falguères et al. 1999) y Sima del Elefante-TE9 (Duval et al. 2009), yacimientos biocronológicamente cercanos al de Vallparadís.

Para un período tan antiguo (>700 ka), el parámetro más problemático a tener en cuenta es la descripción de la incorporación del uranio dentro de los tejidos dentales (13), por su directa relación con los datos isotópicos medidos. Las edades $U$-Th calculadas mediante el programa Isoplot 3.00 (Ludwig 2003) varían entre 226 y 293 ka para el diente EVT0601 y entre 288 y $477 \mathrm{ka}$ para EVT0602 (Fig. 8). Estos resultados demuestran así la ausencia aparente de lixiviación de uranio de estos tejidos y sugieren la aplicación normal del modelo US para ambos casos. Sin embargo, como indica Grün (2009b), el programa DATA presenta ciertas limitaciones cuando la re-

(13) Véase nota 6. lación isotópica ${ }^{230} \mathrm{Th} /{ }^{234} \mathrm{U}$ es superior a 1,05. Habitualmente un valor tan alto indica un fenómeno de lixiviación del uranio, lo que impide la aplicación del modelo US de Grün et al. (1988), pero en el caso de muestras antiguas $(>250 \mathrm{ka})$ tal valor se puede situar por debajo del equilibrio secular si la razón isotópica ${ }^{234} \mathrm{U} / 238 \mathrm{U}$ asociada es muy superior a 1 (detalles en Ku 1976). Es claramente lo que ocurre para EVT0602. Se obtuvieron valores de ${ }^{230} \mathrm{Th} / 234 \mathrm{U}$ en la dentina y el cemento muy superiores a $1(1,115$ y 1,191 , respectivamente, Tab. 2). Ello impide el cálculo de edad ESR-US combinada con el programa DATA aunque, como se ha señalado, no muestran lixiviación aparente

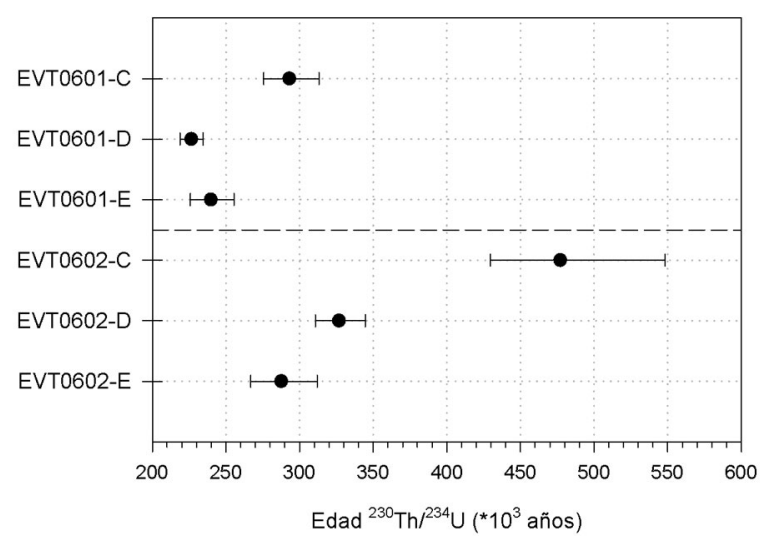

Fig. 8. Edades $U$-Th calculadas mediante el programa Isoplot 3.00 (Ludwig 2003) para todos los tejidos de los dientes de équidos EVT0601 y EVT0602. (C): cemento, (D): dentina, (E): esmalte. 
debido al elevado valor de ${ }^{234} \mathrm{U} / 238 \mathrm{U}(1,75-2,1)$. Por lo tanto, sólo se puede calcular una edad combinada para la muestra EVT0601 con un resultado de 0,83 $\pm 0,07 \mathrm{Ma}$. Para la muestra EVT0602 se ha calculado una edad ESR-EU (es decir, considerando un sistema cerrado para todos los tejidos dentales), con un resultado de $0,54 \pm 0,04 \mathrm{Ma}$ (Tab. 4). Este valor debe considerarse como una edad mínima ya que corresponde a las dosis máximas que se pueden modelizar para los tres tejidos dentales (Fig. 3).

Las 4 muestras de cuarzo datadas por ESR presentan tasas de blanqueo parecidas (entre $56 \mathrm{y}$ $60 \%$ ) (Tab. 5), lo que probablemente indica condiciones similares de depósito y blanqueo. La elección de la función de ajuste es crucial para el cálculo de la dosis equivalente de estas muestras (Duval et al. 2009). En el caso del cuarzo, la utilizada normal (o exclusivamente) en la bibliografía es una función SSE (por ejemplo Rink et al. 2007; Liu et al. 2010; Voinchet et al. 2010). Sin embargo, su uso en las muestras de Vallparadís plantea algunas dificultades: (1) apenas se ajusta a los datos experimentales, algo especialmente evidente en las dosis de radiación bajas (véanse ampliaciones en la Fig. 7) y (2) las curvas nunca pasan por el punto natural. Esto tiene una influencia directa sobre el valor de $D_{E}$ calculado, ya que modifica la pendiente de la curva así determinada, siendo ésta un parámetro fundamental en el proceso de extrapolación. Por este motivo se utilizó una ponderación $1 / \mathrm{I}^{2}$ puesto que da más importancia a las dosis bajas (una discusión sobre la ponderación de los puntos experimentales en luminiscencia y ESR en Berger y Huntley 1986; Grün y Rhodes 1992; Grün y Brumby 1994).

Considerando todo esto, la elección de una función diferente que describiese mejor la evolución de los puntos experimentales fue imprescindible, pero siempre teniendo en cuenta que esta nueva función correspondiese igualmente a un proceso físico. Se eligió empíricamente la que asociara un término exponencial y otro lineal $(E X P+L I N)$ para describir dos características de las muestras de Vallparadís: (1) la presencia de un punto de inflexión alrededor de $6300 \mathrm{~Gy}$, particularmente visible en las muestras de Q1 y Q4 (Fig. 7), lo que indica un cambio en el comportamiento de la señal ESR más allá de este valor de dosis, y (2) el crecimiento continuo de la señal $E S R$ a dosis altas (>10 kGy) sin saturación aparente. La función SSE no tiene en cuenta estos dos fenómenos observados mientras que la función $E X P+L I N$ permite apreciar un cambio de comportamiento de la señal conforme las dosis aplicadas aumentan siendo, en este momento, la función lineal la que define esta parte final de la curva. Este término lineal puede considerarse como una primera aproximación de un término exponencial cuya saturación se encuentra a dosis mucho más altas que la dosis máxima de radiación aplicada. Por lo tanto, la función $E X P+L I N$ permite valorar la hipótesis de una señal ESR asociada al centro Al que presenta dos componentes: una parte dominante correspondiente a las dosis más bajas y con un rápido crecimiento y otra de crecimiento más lento observable en las dosis más elevadas. Estas dos componentes, que podrían ser atribuidas en primera aproximación a trampas que presentan energías de activación aparentemente diferentes, pueden recordar lo que se observa durante el blanqueo óptico. En este caso, la disminución observada puede ser descrita también por dos componentes (Voinchet et al. 2003). Además, comparando los resultados obtenidos con la función $E X P+L I N$ y la $S S E$, se aprecia claramente que el ajuste global de la primera es mucho mejor que el de la segunda (véanse valores de $\mathrm{R}^{2}$ y $\mathrm{X}^{2}$ de la Tab. 5). Esto es evidente en el caso de la pendiente al origen (Fig. 7). Las consecuencias sobre los valores de $D_{E}$ determinados son importantes, ya que los calculados mediante la función $E X P+L I N$ son sistemática y significativamente más bajos que los obtenidos a través de la función SSE (Tab. 5).

Las dosis anuales determinadas para las 3 muestras del sondeo 2, EVT-Q1 a Q3, son del mismo orden de magnitud, entre 2468 y 2619 $\mu \mathrm{Gy} / \mathrm{a}$, mientras que la obtenida para la muestra EVT-Q4 es notablemente inferior, con 1554 $\mu \mathrm{Gy} / \mathrm{a}$ (Tab. 6). Las 4 muestras presentan una distribución similar de los componentes relativos, con una contribución de la dosis beta de más del $50 \%$ de la dosis total frente a menos del $5 \%$ de las dosis alfa y cósmica.

Las edades calculadas son coherentes, ya que las muestras EVT-Q1 a Q3, procedentes del nivel 12, tienen edades $E S R$ de 0,77 $\pm 0,23 \mathrm{Ma}$, $0,78 \pm 0,16$ Ma y $0,83 \pm 0,27$ Ma respectivamente. Sin embargo, teniendo en cuenta los márgenes de error asociados, no es posible distinguir cronológicamente estas muestras de la cuarta EVT-Q4 $(0,83 \pm 0,24 \mathrm{Ma})$ cuya posición estratigráfica está más alta en la secuencia (nivel 10, unidad 7). 


\section{CONCLUSIÓN}

En este estudio se dataron por ESR-US y ESR respectivamente 2 dientes y 4 muestras de sedimentos procedentes de 2 niveles arqueológicos del yacimiento de Vallparadís. Las edades obtenidas a partir del diente EVT0601 y de los cuarzos EVT-Q1 a Q4 son coherentes entre sí en torno a los 0,8 Ma. Los datos son altamente reproductibles, pudiéndose calcular edades promedias sólidas y fiables. A partir de las muestras EVT0601 y EVT-Q4 se obtuvo una edad ESR promedia (promedio ponderado por los errores asociados, con el programa Isoplot 3.00, Ludwig 2003) de 0,83 \pm $0,13 \mathrm{Ma}(2 \sigma)$ para el nivel arqueológico 10 (unidad geológica 7) de la secuencia de Vallparadís (Fig. 9). Las muestras EVT-Q1 a Q3 permiten determinar una edad ESR promedia de 0,79 \pm 0,23 Ma $(2 \sigma)$ para el nivel arqueológico 12 (unidad geológica 8), situado estratigráficamente bajo el nivel 10. Teniendo en cuenta los márgenes de error, estos resultados ESR son consistentes y permiten posicionar el yacimiento cerca de la transición Pleistoceno inferior-medio. Refuerzan los datos iniciales obtenidos a partir del estudio de la microfauna y del paleomagnetismo, ofreciendo así un marco cronoestratigráfico muy completo. La combinación de todos los resultados cronológicos permite atribuir las magnetozonas $\mathrm{N} 1$ y N2 respectivamente a los eventos magnéticos Jaramillo y Brunhes, lo que encuadra cronológicamente su nivel arqueológico 10, localizado en

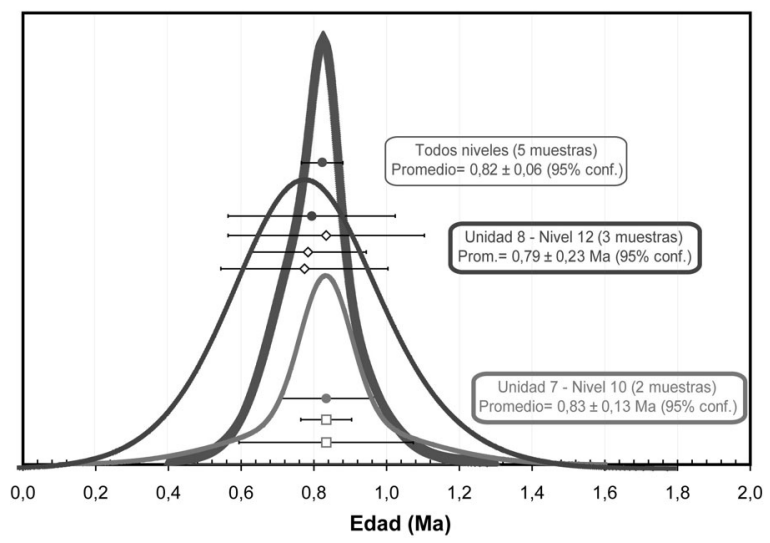

Fig. 9. Yacimiento de Vallparadís (Terrassa, Barcelona): edades ESR promedias obtenidas (calculados mediante el programa Isoplot 3.00, Ludwig 2003). Claves: símbolos sin colorear $=$ edades de las muestras ESR, símbolos llenos $=$ edades promedias . una posición intermedia en la magnetozona inversa R1. La restricción de las edades ESR por los límites Brunhes-Matuyama (0,78 Ma, Gradstein et al. 2004) y Matuyama-Jaramillo (0,99 Ma) indica así que la edad del nivel arqueológico $10 \mathrm{se}$ sitúa entre 0,78 y 0,96 Ma y el nivel 12 entre 0,78 y $0,99 \mathrm{Ma}$. La edad ESR promedia sobre las 5 muestras, y restringida por los datos paleomagnéticos, de 0,83 $\pm 0,05$ Ma parece así la mejor estimación cronológica para el yacimiento de Vallparadís. Estos resultados lo aproximan cronológicamente al nivel TD-6 de Gran Dolina (Falguères et al. 1999), aunque la biocronología de Vallparadís sugiere por su parte una cronología un poco más antigua, anterior a los niveles TD4 de Gran Dolina y cerca del nivel TE9 de Sima del Elefante (Martínez et al. 2010). De todos modos, todos estos datos concuerdan en situar la ocupación en el Pleistoceno inferior final, un período clave sobre el que no existía mucha información disponible hasta el momento. Vallparadís se convierte así en un yacimiento clave para el conocimiento de las primeras ocupaciones humanas de Europa.

\section{AGRADECIMIENTOS}

Queremos agradecer a Norbert Mercier (Centre de Recherche en Physique Appliquée à l'Archéologie, Universidad de Burdeos, Francia) la lectura de los dosímetros TL. El espectrómetro ESR EMX Bruker del Département de Préhistoire du Muséum National d'Histoire Naturelle (Francia) fue financiado por el programa Sésame Ille-de-France. Durante la realización de este trabajo Davinia Moreno ha disfrutado de una beca predoctoral del programa FPI del Ministerio de Ciencia e Innovación (MICINN) (BES-2007-16382).

\section{BIBLIOGRAFÍA}

Adamiec, G. y Aitken, M.J. 1998: "Dose-rate conversion factors: update". Ancient TL 16: 37-50.

Agustí, J. y Madurell, J. 2003: "Los arvicólidos (Muroidea, Rodentia, Mammalia) del Pleistoceno inferior de Barranco León y Fuente Nueva 3 (Orce, Granada). Datos preliminares". En I. Toro, J. Agustí y B. Martínez-Navarro (eds.): El Pleistoceno inferior de Barranco León y Fuente Nueva 3, Orce (Granada). Memoria científica campañas 1999. 
2002. Junta de Andalucía. Consejería de Cultura. Sevilla: 137-146.

Agustí, J.; Oms, O. y Pares, J. M. 2007: "Biostratigraphy, paleomagnetism and geology of the Orce ravine (Southern Spain). Comment on the paper by Gibert et al. (2006)". Quaternary Science Reviews 26: 568-572.

Aitken, M. 1985: Thermoluminescence Dating. Academic Press. London.

Alba, D.; Aurell, J.; Madurell, J.; Gómez, M.; Moyà-Solà, S. y Berasategui, X. 2008: "Paleontologia i geologia del jaciment del Pleistocè inferior de Vallparadís (Terrassa, Vallès Occidental)". Tribuna d'arqueologia 2007: 29-44.

Alberdi, M. T.; Alonso, M. A.; Azanza, B.; Hoyos, M. y Morales, J. 2001: "Vertebrate taphonomy in circum-lake environments: three cases in the Guadix-Baza Basin (Granada, Spain)". Palaeogeography, Palaeoclimatology, Palaeoecology 165: $1-26$.

Arzarello, M.; Marcolini, F.; Pavia, G.; Pavia, M.; Petronio, C.; Petrucci, M.; Rook, L. y Sardella, R. 2007: "Evidence of earliest human occurrence in Europe: the site of Pirro Nord (Southern Italy)". Naturwissenschaften 94: 107-112.

Bahain, J.-J.; Yokoyama, Y.; Falguères, C. y Sarcia, M. N. 1992: "ESR dating of tooth enamel: a comparison with K-Ar dating". Quaternary Science Reviews 11: 245-250.

Berger, G. W. 1990: "Regression and error analysis for a saturating-exponential-plus-linear model". Ancient TL 8: 23-25.

Berger, G. W. y Huntley, D. J. 1986: "Linear regression of TL data". Ancient TL 4: 26-29.

Berger, G. W.; Pérez-González, A.; Carbonell, E.; Arsuaga, J. L.; Bermúdez de Castro, J.-M. y Ku, T. L. 2008: "Luminescence chronology of cave sediments at the Atapuerca paleoanthropological site, Spain". Journal of Human Evolution 55: 300-311.

Bischoff, J.; Rosenbauer, R.; Tavoso, A. y Lumley, H. de. 1988: "A test of uranium-series dating of fossil tooth enamel: Results from Tournal cave, France". Applied Geochemistry 3: 135-141.

Blackwell, B.; Porat, N.; Schwarcz, H. y Debenath, A. 1992: "ESR dating of tooth enamel - comparison with Th-230/U-234 speleothem dates at La-Chaise-de-Vouthon (Charente), France". Quaternary Science Reviews 11: 231-244.

Brennan, B. J. 2003: "Beta doses to spherical grains". Radiation Measurements 37: 299-303.

Brennan, B. J.; Lyons, R. G. y Phillips, S. W. 1991: "Attenuation of alpha particle track dose for spherical grains". Nuclear Tracks and Radiation Measurements 18: 249-253.

Carbonell, E.; Bermúdez de Castro, J.; Pares, J.; Pérez-González, A.; Cuenca-Bescos, G.; Olle, A.; Mosquera, M.; Huguet, R.; Made, J. v. d.; Rosas,
A.; Sala, R.; Vallverdu, J.; García, N.; Granger, D.; Martinon-Torres, M.; Rodríguez, X.; Stock, G.; Verges, J.; Allue, E.; Burjachs, F.; Cáceres, I.; Canals, A.; Benito, A.; Díez, C.; Lozano, M.; Mateos, A.; Navazo, M.; Rodríguez, J.; Rosell, J. y Arsuaga, J. 2008: "The first hominin of Europe". Nature 452: 465-470.

Carbonell, E. y Rodríguez, X. P. 2006: "The first human settlement of Mediterranean Europe". C.R. Palevol 5: 291-298.

Crochet, J.-Y.; Welcomme, J.-L.; Ivorra, J.; Ruffet, G.; Boulbes, N.; Capdevila, R.; Claude, J. y Firmat, C.; Métais, G.; Michaux, J. y Pickford, M. 2009: "Une nouvelle faune de vertébrés continentaux, associée à des artefacts dans le Pléistocène inférieur de l'Hérault (Sud de la France), vers 1,57 Ma". Comptes Rendus Palevol 8: 725-736.

Dauphin, Y. y Williams, C.T. 2004: "Diagenetic trends of dental tissues". Comptes Rendus Palevol 3: 583-590.

Despriée, J.; Gageonnet, R.; Voinchet, P.; Bahain, J. J.; Falguères, C.; Varache, F.; Courcimault, G. y Dolo, J. M. 2006: "Une occupation humaine au Pléistocène inférieur sur la bordure nord du Massif central". Comptes Rendus Palevol 5: 821-828.

Despriée, J.; Voinchet, P.; Tissoux, H.; Moncel, M.-H.; Arzarello, M.; Robin, S.; Bahain, J.-J.; Falguères, C.; Courcimault, G.; Dépont, J.; Gageonnet, R.; Marquer, L.; Messager, E.; Abdessadok, S. y Puaud, S. 2010: "Lower and middle Pleistocene human settlements in the Middle Loire River Basin, Centre Region, France". Quaternary International 223-224: 345-359.

Dolo, J. M.; Lecerf, N.; Mihajlovic, V.; Falguères, C. y Bahain, J.-J. 1996: "Contribution of ESR dosimetry for irradiation of geological and archaeological samples with a 60-Co panoramic source". Applied Radiation and Isotopes 47: 1419-1421.

Driessens, F. C. M. 1980: "The mineral in bone, dentine and tooth enamel". Bulletin de la Société Chimique de Belgique 89: 663-689.

Duttine, M.; Villeneuve, G.; Bechtel, F. y Demazeau, G. 2002: "Caractérisation par résonance paramagnétique électronique (RPE) de quartz naturels issus de différentes sources". Comptes Rendus Geoscience 334: 949-955.

Duval, M.; Grün, R.; Falguères, C.; Bahain, J.-J. y Dolo, J.-M. 2009: “ESR dating of Lower Pleistocene fossil teeth: limits of the single saturating exponential (SSE) model for the equivalent dose determination". Radiation Measurements 44: 477-482.

Falguères, C.; Bahain, J.-J.; Duval, M.; Shao, Q.; Han, F.; Lebon, M.; Mercier, N.; Pérez-González, A.; Dolo, J.-M. y García, T. 2010: "A 300-600 ka ESR/U-series chronology of Acheulian sites in Western Europe". Quaternary International 223224: 293-298. 
Falguères, C.; Bahain, J.-J.; Yokoyama, Y.; Arsuaga, J. L.; Bermúdez de Castro, J. M.; Carbonell, E.; Bischoff, J. L. y Dolo, J. M. 1999: "Earliest humans in Europe: the age of TD6 Gran Dolina, Atapuerca, Spain". Journal of Human Evolution 37: 343-352.

Gradstein, F.; Ogg, J. y Smith, A. 2004: A geologic time scale. Cambridge University Press. Cambridge.

Granger, D. E. y Muzikar, P. F. 2001: "Dating sediment burial with in situ-produced cosmogenic nuclides: theory, techniques, and limitations". Earth and Planetary Science Letters 188: 269-281.

Grün, R. 1989: "Electron spin resonance (ESR) dating”. Quaternary International 1: 65-109.

Grün, R. 2000: "Methods of dose determination using ESR spectra of tooth enamel". Radiation Measurements 32: 767-772.

Grün, R. 2007: "Electron spin resonance dating". En S. A. Elias (ed.): Encyclopedia of Quaternary Science. Elsevier. Amsterdam: 1505-1516.

Grün, R. 2009a: "The relevance of parametric U-uptake models in ESR age calculations". Radiation Measurements 44: 472-476.

Grün, R. 2009b: "The DATA program for the calculation of ESR age estimates on tooth enamel". Quaternary Geochronology 4: 231-231.

Grün, R. y Brumby, S. 1994: "The assessment of errors in past radiation doses extrapolated from ESR/TL dose-response data". Radiation Measurements 23: 307-315.

Grün, R. y Katzenberger-Apel, O. 1994: “An alphairradiator for ESR dating”. Ancient TL 12: 35-38.

Grün, R. y McDermott, F. 1994. "Open system modelling for U-series and ESR dating of teeth". Quaternary Science Reviews (Quaternary Geochronology) 13: 121-125.

Grün, R. y Rhodes, E. J. 1991: “On the selection of dose points for saturating exponential ESR/TL dose response curve". Ancient TL 9: 40-46.

Grün, R. y Rhodes, E. J. 1992: "Simulations of saturating exponential ESR/TL dose response curves weighting of intensity values by inverse variance". Ancient TL 10: 50-56.

Grün, R.; Schwarcz, H. P. y Chadam, J. 1988: “ESR dating of tooth enamel: coupled correction for U-uptake and U-series desiquilibrium". Nuclear Tracks Radiation Measurements 14: 237-244.

Guadelli, J.; Sirakov, N.; Ivanova, S.; Sirakova, S.; Anastassova, E.; Courtaud, P.; Dimitrova, I.; Djabarska, N.; Fernández, P.; Ferrier, C.; Fontugne, M.; Gambier, D.; Guadelli, A.; Iordanova, D.; Iordanova, N.; Kovatcheva, M.; Krumov, I.; Leblanc, J.-C.; Mallye, J.-B.; Marinska, M.; Miteva, V.; Popov, V.; Spassov, R.; Taneva, S.; Tisterat-Laborde, N. y Tsanova, T. 2005: "Une séquence du Paleolithique Inferieurr au Paleolithique Récent dans les Balkans: la grotte Kozarnika á Orechets (nord-ouest de la Bulgarie)". En N. Molines, M.-H. Moncel y J.-L. Monnier (eds.): Les Premiers Peuplements en Europe. British Archaeological Reports, International Series 164. Archaeopress. Oxford: 87-103.

Hillson, S. 2005: Teeth. Cambridge University Press. Cambridge.

Ikeya, M. 1982: “A model of linear uranium accumulation for ESR age of Heidelberg (Mauer) and Tautavel bones". Japanese Journal of Applied Physics 21: 690-692.

Ikeya, M. 1993: New applications of electron spin resonance - dating, dosimetry and microscopy. World Scientific Publishing. Singapore.

Kahlke, R.-D. y Gaudzinski, S. 2005: "The blessing of a great flood: differentiation of mortality patterns in the large mammal record of the Lower Pleistocene fluvial site of Untermassfeld (Germany) and its relevance for the interpretation of faunal assemblages from archaeological sites". Journal of Archaeological Science 32: 1202-1222.

Kohn, M. J.; Schoeninger, M. J. y Barker, W. W. 1999: "Altered states: Effects of diagenesis on fossil tooth chemistry". Geochimica and Cosmochimica Acta 63: 2737-2747.

$\mathrm{Ku}$, T. L. 1976. "The uranium-series methods of age determination". Annual Review of Earth and Planetary Sciences 4: 1-70.

Laurent, M.; Falguères, C.; Bahain, J.-J.; Rousseau, L. y Van Vliet Lanoé, B. 1998: "ESR dating of quartz extracted from Quaternary and neogene sediments: method, potential and actual limits". Quaternary Geochronology 17: 1057-1062.

Lin, M.; Yin, G.; Ding, Y.; Cui, Y.; Chen, K.; Wu, C. y Xu, L. 2006: "Reliability study on ESR dating of the aluminium center in quartz". Radiation Measurements 41: 1045-1049.

Liu, C. R.; Yin, G.-M.; Gao, L.; Bahain, J.-J.; Li, J.-P.; Lin, M. y Chen, S.-M. 2010: "ESR dating of Pleistocene archaeological localities of the Nihewan Basin, North China - Preliminary results" Quaternary Geochronology 5: 385-390.

Lordkipanidze, D.; Jashashvili, T.; Vekua, A.; Ponce de León, M. S.; Zollikofer, C. P. E.; Rightmire, G. P.; Pontzer, H.; Ferring, R.; Oms, O.; Tappen, M.; Bukhsianidze, M.; Agustí, J.; Kahlke, R.; Kiladze, G.; Martínez-Navarro, B.; Mouskhelishvili, A.; Nioradze, M. y Rook, L. 2007: "Postcranial evidence from early Homo from Dmanisi, Georgia". Science 449: 305-310.

Lumley, H. de; Barsky, D. y Cauche, D. 2009: “Les premières étapes de la colonisation de l'Europe et l'arrivée de l'Homme sur les rives de la Méditerranée". L'Anthropologie 113: 1-46.

Lumley, H. de; Fournier, A.; Krzepkowska, J. y Echassoux, A. 1988: "L'industrie du Pléistocène infé- 
rieur de la grotte du Vallonnet (Roquebrune-CapMartin, Alpes-Maritimes". L'Anthropologie 92: 501-614.

Ludwig, K. R. 2003: User's Manual for Isoplot 3.00. Manual. Berkeley Geochronology Center. Berkeley, CA.

Marfunin, A. 1979: Spectroscopy, luminescence and radiation centers in minerals, Springer-Verlag Berlin Heidelberg.

Martínez, K.; García, J.; Carbonell, E.; Agustí, J.; Bahain, J.-J.; Blain, H.-A.; Burjachs, F.; Cáceres, I.; Duval, M.; Falguères, C.; Garcés, M.; Gómez, M. y Huguet, R. 2010: "A new Lower Pleistocene archaeological site in Europe: Vallparadís (Barcelona, Spain)". Proceedings of the National Academy of Sciences of the United States of America 107 (13): 5762-5767.

Martínez-Navarro, B.; Espigares, M. P. y Ros, S. 2003: "Estudio preliminar de las asociaciones de grandes mamíferos de Fuente Nueva-3 y Barranco León-5 (Orce, Granada, España)". En I. Toro, J. Agustí y B. Martínez-Navarro (eds.): El Pleistoceno inferior de Barranco León y Fuente Nueva 3, Orce (Granada). Memoria científica campañas 1999-2002. Junta de Andalucía. Consejería de Cultura. Sevilla: 115-136.

Martínez-Navarro, B.; Turq, A.; Agustí Ballester, J. y Oms, O. 1997: "Fuente Nueva-3 (Orce, Granada, Spain) and the first human occupation of Europe". Journal of Human Evolution 33: 611-620.

Mercier, N. y Falguères, C. 2007: "Field gamma dose-rate measurement with a $\mathrm{NaI}(\mathrm{Tl})$ detector: re-evaluation of the 'threshold' technique". Ancient TL 25: 1-4.

Murray, A. S. y Roberts, R. G. 1997: "Determining the burial time of single grains of quartz using optically stimulated luminescence". Earth and Planetary Science Letters 152: 163-180.

Oms, O.; Pares, J. M.; Martínez-Navarro, B.; Agustí, J.; Toro, I.; Martínez-Fernández, G. y Turq, A. 2000: "Early human occupation of Western Europe: Paleomagnetic dates for two paleolithic sites in Spain". Proceedings of the National Academy of Sciences of the United States of America 97: 10666-10670.

Palombo, M. y Ferretti, M. 2005: "Elephant fossil record from Italy: knowledge, problems, and perspectives". Quaternary International 126-128: 107-136.

Palombo, M.; Sardella, R. y Novelli, M. 2008: "Carnivora dispersal in Western Mediterranean during the last 2.6Ma". Quaternary International 179: 176-189.

Parés, J. y Pérez-González, A. 1999: "Magnetochronology and stratigraphy at Gran Dolina section, Atapuerca (Burgos, Spain)". Journal of Human Evolution 37: 325-342.
Peretto, C.; Amore, F. O.; Antoniazzi, Alb.; Antoniazzi, Ald.; Bahain, J. J.; Cattani, L.; Cavallini, E.; Esposito, P.; Falguères, C.; Gagnepain, J.; Hedley, I.; Laurent, M.; Lebreton, V.; Longo, L.; Milliken, S.; Monegati, P.; Ollé, A.; Pugliese, N.; Renault-Miskovsky, J.; Sozzi, M.; Ungaro, S.; Vannucci, S.; Verges, J. M.; Wagner, J. J. y Yokoyama, Y. 1998: "L'industrie lithique de Ca'Belvedere di Monte Poggiolo: Stratigraphie, matière première, typologie, remontages et traces d'utilisation'. L'Anthropologie 102: 343-465.

Piepenbrink, H. 1989: "Examples of chemical changes during fossilisation". Applied Geochemistry 4: 273-280.

Postigo Mijarra, J. M.; Burjachs, F.; Gómez Manzaneque, F. y Morla, C. 2007: "A palaeoecological interpretation of the lower-middle Pleistocene Cal Guardiola site (Terrassa, Barcelona, NE Spain) from the comparative study of wood and pollen samples". Review of Palaeobotany and Palynology 146: 247-264.

Prescott, J. R. y Hutton, J. T. 1988: "Cosmic ray and gamma ray dosimetry for TL and ESR". Nuclear Tracks Radiation Measurements 14: 223-227.

Prescott, J. R. y Hutton, J. T. 1994: "Cosmic ray contributions to dose rates for luminescence and ESR dating: large depths and long-term time variations". Radiation Measurements 23: 497-500.

Preusser, F.; Chithambo, M.; Götte, T.; Martini, M.; Ramseyer, K.; Sendezera, E.; Susino, G. y Wintle, A. 2009: "Quartz as a natural luminescence dosimeter". Earth-Science Reviews 97: 184-214.

Rhodes, E. J.; Singarayer, J. S.; Raynal, J. P.; Westaway, K. E. y Sbihi-Alaoui, F. Z. 2006: "New age estimates for the Palaeolithic assemblages and Pleistocene succession of Casablanca, Morocco". Quaternary Science Reviews 25: 2569-2585.

Rink, W. J. 1997: "Electron spin resonance (ESR) dating and ESR applications in Quaternary science and archaeometry". Radiation Measurements 27: 975-1025.

Rink, W. J.; Bartoll, J.; Schwarcz, H. P.; Shane, P. y Bar-Yosef, O. 2007: "Testing the reliability of ESR dating of optically exposed buried quartz sediments". Radiation Measurements 42: 1618-1626.

Rodríguez, J. 2001: "Structure de la communauté de mammifères pléistocènes de Gran Dolina (Sierra de Atapuerca, Burgos, Espagne)". L'Anthropologie 105: 131-157.

Rook, L. y Martínez-Navarro, B. 2010: "Villafranchian: The long story of a Plio-Pleistocene European large mammal biochronologic unit". Quaternary International 219: 134-144.

Szabo, B. 1979: "Dating Fossil Bone from Cornelia, Orange Free State, South Africa". Journal of Archaeological Science 6: 201-203. 
Toyoda, S. y Falguères, C. 2003: "The method to represent the ESR signal intensity of the aluminium hole center in quartz for the purpose of dating". Advances in ESR Applications 20: 7-10.

Toyoda, S. e Ikeya, M. 1991: “Thermal stabilities of paramagnetic defects and impurity centers in quartz: basis for ESR dating of thermal history". Geochemical Journal 25: 437-445.

Vandenberghe, D.; De Corteb, F.; Buylaert, J. P.; Kucera, J. y Van den Haute, P. 2008: "On the internal radioactivity in quartz". Radiation Measurements 43: 771-775.

Voinchet, P.; Bahain, J.-J.; Falguères, C.; Laurent, M.; Despriée, J.; Gageonnet, R. y Chaussé, C. 2004: "ESR dating of quartz extracted from Quaternary sediments: application to fluvial terraces system of nothern France". Quaternaire 15: 135-141.

Voinchet, P.; Despriée, J.; Tissoux, H.; Falguères, C.; Bahain, J.-J.; Gageonnet, R.; Dépont, J. y Dolo, J.-M. 2010: "ESR chronology of alluvial deposits and first human settlements of the Middle Loire Basin (Region Centre, France)". Quaternary Geochronology 5: 381-384.

Voinchet, P.; Falguères, C.; Laurent, M.; Toyoda, S.; Bahain, J.-J. y Dolo, J.-M. 2003: “Artificial optical bleaching of the aluminium center in quartz impli- cations to ESR dating of sediments". Quaternary Science Reviews 22: 1335-1338.

Voinchet, P.; Falguères, C.; Tissoux, H.; Bahain, J. J.; Despriée, J. y Pirouelle, F. 2007: "ESR dating of fluvial quartz: estimate of the minimal distance transport required for getting a maximum optical bleaching". Quaternary Geochronology 2: 363366.

Weil, J. A. 1984: "A review of electron spin spectroscopy and its application to the study of paramagnetic defects in crystalline quartz". Physics and Chemistry of Minerals 10: 149-165.

Yokoyama, Y.; Bibron, R. y Falguères, C. 1988: “Datation absolue des planchers stalagmitiques de la grotte du Vallonnet à Roquebrune-Cap-Martin (Alpes-Maritimes, France), par la résonance de spin électronique (ESR)'”. L'Anthropologie 92: 429-436.

Yokoyama, Y.; Falguères, C. y Quaegebeur, J. P. 1985: "ESR dating of quartz from Quaternary sediments: first attempts". Nuclear Tracks 10: 921-928.

Yokoyama, Y. y Nguyen, H. V. 1980: "Direct and non destructive dating of marine sediments, manganese nodules and corals by high resolution gamma-ray spectrometry". En E. D. Goldberg, Y. Horibe y K. Saruhashi (eds.): Isotope Marine Chemistry. Uchida Rokakuho, Tokyo: 259-289. 